\title{
COVERAGE DEPENDENCE OF THE SPIN-RESOLVED PHOTOEMISSION FROM EPITAXIALLY GROWN Ag LAYFRS ON Pt(11)
}

\author{
B. SCHMIEDESKAMP, B. KESSLER, B. VOGT and U. HEINZMANN \\ Fakultät für Physik, Universität Bielefeld, D-4800 Bielefeld, Fed. Rep. of Germany \\ and \\ Fritz-Haber-Institut der Max-Planck-Gesellschaft, D-1000 Berlin 33, Germany
}

Received 19 April 1989; accepted for publication 5 July 1989

$\mathrm{Ag} / \mathrm{Pt}(111)$ has been studied by spin-, angle-, and energy-resolved photoemission with circularly polarized synchrotron radiation of BESSY for different $\mathrm{Ag}$ coverages. The prepared layers were characterized by AES and LEED and turned out to grow epitaxially. An independent layer thickness determination and some additional structure information were obtained from Rutherford backscattering studies in combination with channeling. The spin-resolved photoemission experiments were performed for normal incidence of circularly polarized light and normal emission of the photoelectrons. The spin information allowed a separation of spin-orbit split peaks of the Ag adsorbate layer and partly also a separation of $\mathrm{Ag}$ adsorbate peaks from the $\mathrm{Pt}$ substrate background intensity. This "method-induced improved resolution" was used to deduce information about the development of the $\mathrm{Ag}$ band structure in the $\Lambda$-direction from the coverage dependence of spin-resolved photoemission spectra. Dispersion of occupied bands was found to be already almost completely developed for a three-layer system. Information about the development of the band structure in its unuccupied part was obtained from the resonant behavior of peaks at about $6 \mathrm{eV}$ below $E_{\mathrm{F}}$. The resonant behavior is already observed for three $\mathrm{Ag}$ layers and increases with layer thickness. We observe a peak in the photoemission spectra contributing to the resonances which is not predicted by existing theoretical models.

\section{Introduction}

Spin-resolved photoemission with circularly polarized radiation has been studied in the past with a couple of nonmagnetic systems. Rare gas adsorbate photoemission [1,2] yielded information about peak splittings, resonances, and symmetries of the levels involved in transitions, while from similar studies with metal [3-5] and insulator single crystals [6] a symmetry resolved band mapping was obtained. 
The adsorbate system $\mathrm{Ag} / \mathrm{Pt}$ has been studied by different experimental methods. TDS, AES, and LEED yielded [7] information about the growth and adsorption energies of $\mathrm{Ag}$ on different Pt surfaces including the (111) surface. Photoemission studies have been performed on $\mathrm{Ag} / \mathrm{Pt}(100)[8]$ as well as on $\mathrm{Ag}(111)[9] \mathrm{Ag}(100)[10]$ and $\mathrm{Pt}(111)[3,9]$ single crystals which gave an insight in the electronic structure of these systems.

In this work we describe (to our knowledge) the first spin-resolved photoemission experiment with a non-ferromagnetic metal adsorbate: $\mathrm{Ag} / \mathrm{Pt}(111)$. For Ag coverages between 1 and 8 layers we observe the development of the three-dimensional band structure in the $\Lambda$-direction. By means of the spin analysis it is possible to reveal details of this development which cannot be obtained from a non-spin-resolved photoemission experiment because of ten the splitting of adsorbate peaks is smaller than the lifetime broadening and substrate and adsorbate peaks have the same energetic position (for example $\mathrm{Ag} / \mathrm{Au}[9,11,12])$. Beyond it, spin-resolved photoemission yields information about hybridization $[5,13]$ and especially about avoided crossings in the band structure $[4,6]$.

\section{Experimental}

The experiments were performed at the $6.5 \mathrm{~m}$ normal incidence VUV monochromator [14] at BESSY (circularly polarized off-plane radiation). The apparatus used for the measurements has been described previously [3], in addition an evaporator was integrated.

All spin-resolved photoemission data were obtained for normal incidence of the circularly polarized radiation and normal emission of the photoelectrons. The photoelectrons were analyzed with respect to their kinetic energy and emission angle by an electron spectrometer [15]. The overall energetic resolution (electrons plus photons) was better than $150 \mathrm{meV}$ at an angular resolution of $\pm 3^{\circ}$.

The surface normal of the $\mathrm{Pt}(111)$ crystal (mounted on top of a manipulator) coincided within $0.5^{\circ}$ with the [111] direction and within $0.3^{\circ}$ with the light direction. The clean crystal surface was prepared by cycles of $\mathrm{Ar}^{+}$and $\mathrm{Ne}^{+}$bombardment, heating in oxygen, and flashing. It was controlled by Auger electron spectroscopy (AES) and LEED.

The evaporation was performed with the substrate at room temperature with a resistively heated $\mathrm{Ag}$ evaporator, surrounded by a cooled shield. It is placed $\sim 20 \mathrm{~cm}$ away from the target. The $\mathrm{Ag}$ beam was geometrically collimated by a small tube. After an initial outgassing the evaporator worked without strong influence on the UHV conditions $\left(<10^{-9}\right.$ mbar during evaporation, base pressure $<3 \times 10^{-10} \mathrm{mbar}$ ). It was used for short periods, between them the surface was controlled by LEED and AES. 


\section{Adsorbate characterization with AES, LEED, photoemission, and RBS/ channeling}

The characterization of the $\mathrm{Ag}$ adsorbates in this work is based on a combination of AES, LEED, RBS and spin-resolved photoemission.

The growth was studied by LEED and AES. Typical results are presented in fig. 1. The upper part shows the LEED spots of the system $\mathrm{Ag} / \mathrm{Pt}(111)$, which turned out to show no change in geometry during the time of evaporation from the clean $\mathrm{Pt}(111)$ crystal up to the maximum time of cvaporation (45 $\mathrm{min}$ ). This behavior was studied for several different electron energies between 50 and $500 \mathrm{eV}$ and did not yield spot splittings.
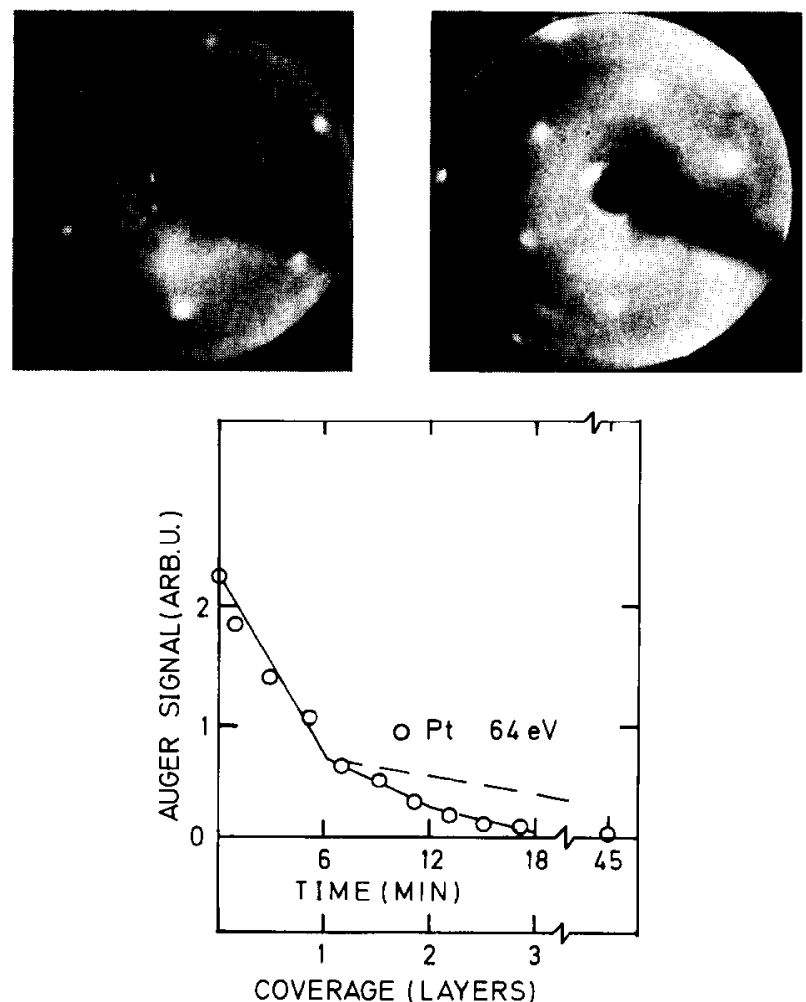

Fig. 1. Characterization of the Ag adsorbate. Upper part: LEED pattern observed for $\operatorname{Pt}(111)$ and $\mathrm{Ag} / \mathrm{Pt}(111)$ up to evaporation times of 45 minutes (coverage $\Theta \approx 8$ layers) left: $90 \mathrm{eV}$, right: 140 $\mathrm{eV}$. Lower part: Auger signal intensities versus evaporation time, ) or $(---)$ are fit curves assuming a layer-by-layer or Stranski Krastanov growth, respectively. The completion of the first layer was obtained by means of the ratio of the $\mathrm{Pt}$ signal for 1 layer and of the clean $\mathrm{Pt}$ surface which is known in the literature [7]. This calibration was confirmed by RBS studies (see below, fig. 3). 
An AST plot (Auger-signal intensities versus evaporation time [16]) is given in the lower part of fig. 1. We take the Pt Auger transition at $64 \mathrm{eV}$ because it arises solely from Pt. The peak heights are taken as measured and are not normalized. The calibration of the coverage versus evaporation time was done by means of an AST plot for $\mathrm{Ag}$ on $\mathrm{Pt}(111)$ given in ref. [7]. As the authors of ref. [7] obtain a Stranski-Krastanov growth for Ag on Pt(111), the AST plot shows a pronounced kink at the saturation of 1 layer. The $64 \mathrm{eV} \mathrm{Pt}$ Auger peak intensity at this coverage is by a factor of 3.3 smaller than for a clean $\mathrm{Pt}$ crystal. Assuming a layer-by-layer growth and making the assumption that the Ag deposited on the crystal is proportional to the evaporation time (number of short evaporation intervals) [17] we obtain the fit curve given as series of solid straight lines in the AST plot of fig. 1. For Ag coverages larger than one layer these data are compared in fig. 1 with the experimental data of ref. [7] which are represented by the dashed curve.

Obviously due to the different way of preparing the layers - we prepared thick Ag layers by several short evaporations with breaks in between - we find no pronounced break in the AST plot at a coverage of 1 layer (the Ag coverage calibration was independently proved by RBS, compare below, fig. 3) and a by far stronger decrease of the $\mathrm{Pt}$ intensity with increasing coverage. Together with the LEED results described above we conclude thus that in our case $\mathrm{Ag}$ grows epitaxially in almost layer-by-layer fashion [18] on $\operatorname{Pt}(111)$ up to coverages of about 8 layers. The epitaxial growth under our conditions may be due to an annealing effect during the breaks.

The symmetry of the adsorbate layer could be determined by non-spin-resolved off-normal photoemission. For these measurements we used normally incident light with a photon energy of $23 \mathrm{eV}$, as for this energy the Pt yield is drastically reduced compared to the $\mathrm{Ag}$ yield. Photoelectrons were collected at an angle of $20^{\circ}$ with respect to the $\mathrm{Pt}$ surface normal. For different rotation angles $\omega$ about the crystal surface normal we obtain the spectra displayed in fig. 2. All the spectra can be separated into a broad secondary electron background which is also observed for a pure $\operatorname{Pt}(111)$ crystal and the sharp peak structures at low binding energy, which are due to silver. Sharp maxima of the $\mathrm{Ag}$ yield are observed for $\omega=60^{\circ}, 180^{\circ}$, and $300^{\circ}$, while the spectra are completely different for $\omega=120^{\circ}$ and $240^{\circ}$. The spectra were obtained for a three-layer Ag adsorbate and show that this layer has already threefold symmetry.

An independent characterization regarding the thickness and the structure of the Ag layer was performed with Rutherford backscattering in combination with channeling. For this experiment a 1.5 layer Ag adsorbate on $\mathrm{Pt}(111)$ was prepared as described above and then transferred to a Rutherford backscattering apparatus, which is, e.g., briefly described in ref. [19]. $280 \mathrm{keV} \mathrm{He}$ ions were used and yield, for ion incidence along a random direction of the $\mathrm{Ag} / \mathrm{Pt}(111)$ system the backscattering spectrum in the lower left part of fig. 3 . 


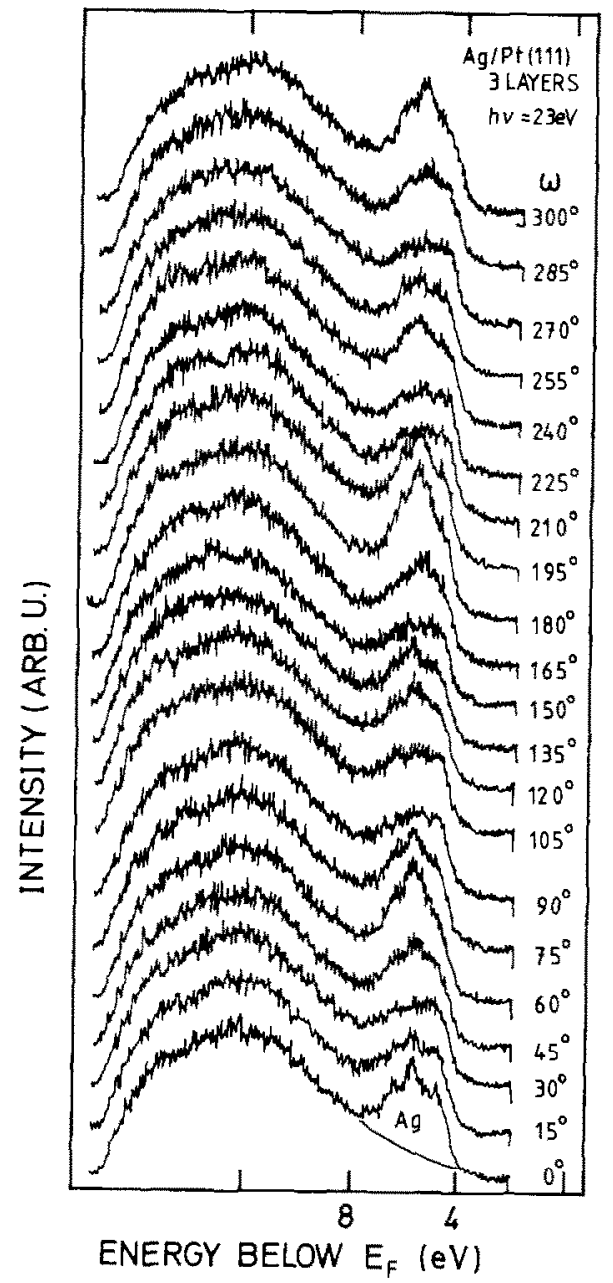

Fig. 2. Characterization of the symmetry for a three-layer $\mathrm{Ag}$ adsorbate by photoemission. The spectra were obtained for $23 \mathrm{eV}$ normally incident photons, an emission angle of $\theta=20^{\circ}$ with respect to the surface normal, and different rotation angles $\omega$ about the surface normal. The symmetry turns out to be threefold.

A straight forward thickness calibration from this spectrum is unfortunately not possible, as the yield of backscattered particles is almost completely due to backscattering from $\mathrm{Pt}$. The small area in the upper right part of the spectrum indicates the yield expected for backscattering from $1.5 \mathrm{Ag}$ layers [20].

For He-ion incidence along a channeling direction the yield for backscattering from the Pt substrate is drastically reduced and we obtain for the [110] direction (i.e. ion incidence at $\phi_{\mathrm{in}}=35.26^{\circ}$ ) and 3 different directions $\phi_{\text {out }}$ of 


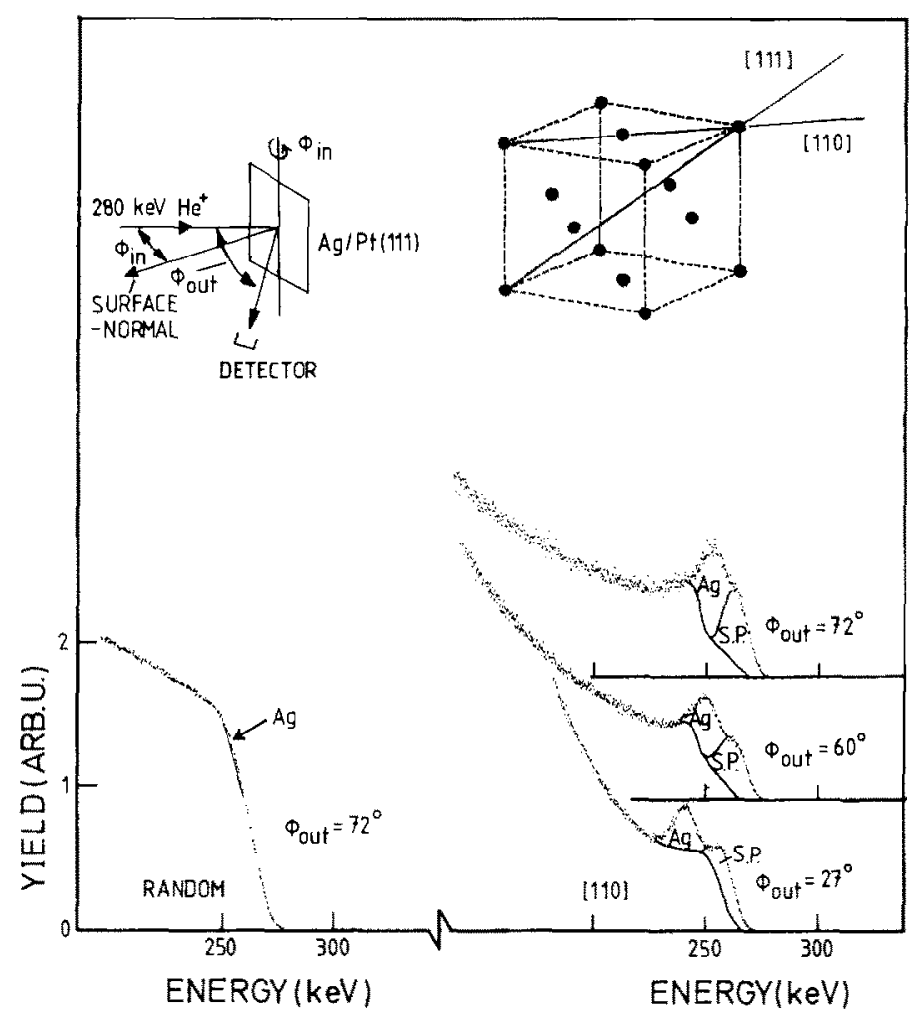

Fig. 3. Characterization of a 1.5 layer Ag adsorbate by RBS/Channeling. Upper part, left: experimental setup. Upper part, right: incidence directions of the ion beam along a [110] and [111] axial channeling direction in a cubic fcc lattice. Lower part, left: Rutherford backscattering (RBS) spectrum for $280 \mathrm{keV} \mathrm{He}$ ion incidence along a random direction in $\mathrm{Ag} / \mathrm{Pt}(111)$. Lower part, right: RBS-spectra for $280 \mathrm{keV} \mathrm{He}{ }^{+}$incidence along the [110] axis and 3 different detection angles.

the outgoing particles the backscattering spectra in the lower right part of fig. 3. They consist of 3 parts: the "surface peak" (S.P.) due to backscattering from $P t$ in the first few layers and a depth dependent yield, which is due to backscattering of those particles in the ion beam, which are not channeled [21] as well as the yield for backscattering from the $\mathrm{Ag}$ adsorbate. The parts can be separated as they show a different behavior with $\phi_{\text {out }}$, the ion detection angle. The Ag peak has a stronger energy shift with $\phi_{\text {out }}$ than the Pt-surface peak, the random yield of a certain depth interval is spread over a larger energy interval with increasing $\phi_{\text {out }}$. Considering these conditions quantitatively we obtain the separation of the spectra as given in fig. 3. The absolute calibration of the spectra was done by means of the random spectrum (left part of the figure) [22]. This procedure yields $1.4 \pm 0.2$ layers of silver and $0.45 \pm 0.1$ layers of $\mathrm{Pt}_{\mathrm{t}}$ corresponding to the surface peak. 
In contrast to this, one would expect at least the yield of 1.1 ML [23] in the surface peak of an unscreened Pt[110] string. The Pt-surface layers are thus effectively screened by the $\mathrm{Ag}$ adsorbate which forms itself a well-ordered commensurate overlayer [24]. The value of $1.4 \pm 0.2$ layers for the $\mathrm{Ag}$ yield is only a lower limit for the real $\mathrm{Ag}$ coverage as screening effects in the $\mathrm{Ag}$ layer itself might have reduced the yield. This cannot occur for incidence along the [111] direction if the total amount of $\mathrm{Ag}$ is lower than $3 \mathrm{ML}$, as in this case at least $3 \mathrm{ML}$ are visible for the ion beam (see fig. 3, upper right part). A similar set of backscattering spectra was therefore also determined for the [111] direction. From this set of data we also obtain $1.4 \pm 0.2 \mathrm{ML} \mathrm{Ag}$ but $2 \pm 0.2$ ML $\mathrm{Pt}$ contributing to the surface peak. It can thus be concluded that the RBS/channeling studies yield $1.4 \pm 0.2 \mathrm{ML} \mathrm{Ag}$ coverage in agreement with the Auger calibration of $1.5 \pm 0.3 \mathrm{ML}$ and that the $\mathrm{Ag}$ layer is well-ordered and largely in positions, which are a direct continuation of the underlying $\mathrm{Pt}$ lattice [25].

\section{Results and discussion}

The $\mathrm{Ag}$ layers prepared in this way were studied with spin-resolved photoemission for different coverages and photon energies. Photoemission spectra for $16 \mathrm{eV}$ photon energy and different $\mathrm{Ag}$ coverages are given in fig. 4 . In each part of the figure the total photoelectron intensities $I$ (pronounced dots) is given and separated into the partial intensities $I_{+}$(dashed curve) and $I_{-}$(solid curve) by means of the spin polarization $P$ according to

$I_{+}=\frac{1}{2} I(1+P), \quad I_{-}=\frac{1}{2} I(1-P)$.

$I_{+}$denotes the intensity of the photoelectrons with spin parallel to the photon spin, $I_{-}$the intensity of photoelectrons with the opposite spin direction.

The spectrum in the lowest part of fig. 4 was obtained for the clean Pt(111) crystal and agrees with a spectrum obtained in ref. [3] where this system has been studied extensively. The four peaks in the spectrum correspond to transitions from the two first spin-orbit split band pairs below $E_{\mathrm{F}}$ into the free electron like final band [3].

For higher coverages the non-spin-resolved spectra (•) show an intensity increase and an energy shift of the peak at about $4.3 \mathrm{eV}$ below $E_{\mathrm{F}}$ towards higher binding energy, while the first 3 peaks decrease rapidly with coverage until they can hardly be resolved for a coverage of about 8 layers. We compare this spectrum for 8 layers with corresponding spectra obtained for a $\mathrm{Ag}(111)$ crystal in ref. [9] and find very good agreement regarding the shape of the spectrum and the intensity peak position $\left(4.7 \mathrm{eV}\right.$ below $\left.E_{\mathrm{F}}\right)$. Further spectra of the thick layer system obtained for photon energies between 11 and $24 \mathrm{eV}$, which are given in a separate publication [26], yield quantitatively the same 


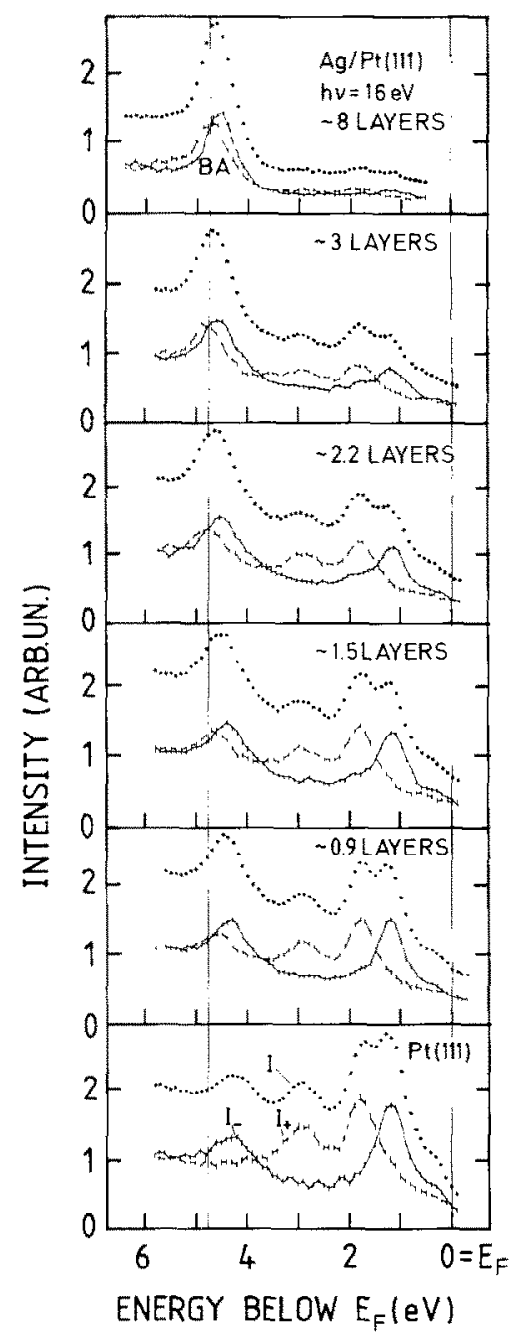

Fig. 4. Photoelectron intensities of $\mathrm{Ag} /$ $\mathrm{Pt}(111)$ for different coverages from $\Theta=0$ up to $\Theta=8$ layers at $h \nu=16 \mathrm{eV}$. The total intensity is marked by points ( $\bullet$, the partial intensities $I_{+}$and $I_{-}$are marked by dashed and solid lines, respectively. The first 3 peaks below $E_{\mathrm{F}}$ correspond to transitions of $\mathrm{Pt}$, the 4th peak contains contributions of $\mathrm{Ag}$ and $\mathrm{Pt}$ (only the $I_{+}$part in the 4 th peak is exclusively due to $\mathrm{Ag}$ ). The error bars show the total error including the statistical errors of the count rates and the uncertainty of light polarization and detector asymmetry function.

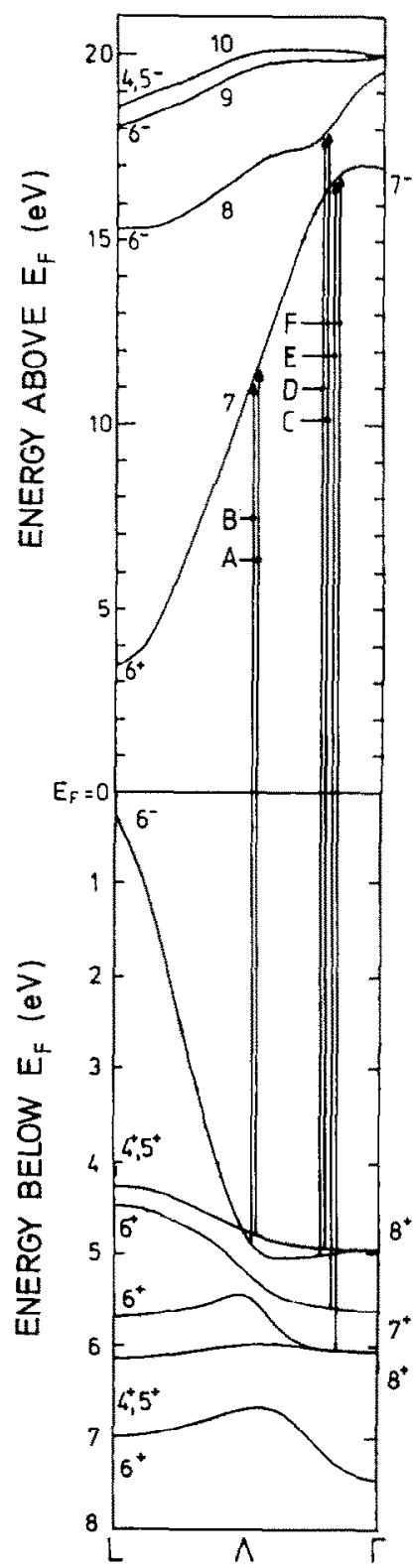

Fig. 5. Bulk band structure of $\mathrm{Ag}$ in the $\Lambda$-direction by Eckhard et al. [27]. A and B indicate the transitions corresponding to the peaks in the upper part of fig. 4, C-F indicate the transitions of peaks $\mathrm{C}-\mathrm{F}$ in the upper part of fig. 7 . 
peak shifts with photon energy as those for the $\operatorname{Ag}(111)$ crystal and support further the conclusion that from the point of view of photoemission an 8 layer epitaxially grown $\mathrm{Ag}$ adsorbate can be regarded as a $\mathrm{Ag}(111)$ crystal. Using the spin-polarization information we make now a further analysis of the spectra. We regard again the peak between 4.3 and $4.7 \mathrm{eV}$ below $E_{\mathrm{F}}$ which is separated into the partial intensities $I_{+}$and $I_{-}$and it can be seen that for all coverages except the clean $\mathrm{Pt}(111)$ crystal the peak consists of two peaks with opposite spin polarization. For low coverages the $I_{-}$peak dominates as a considerable part of the $I_{\text {- }}$ peak corresponds to transitions of the pure $\mathrm{Pt}$ crystal. For higher coverages the Pt part in the spectra decreases and $I_{+}$and $I_{-}$approach equal peak intensities. For the $\mathrm{Ag}(111)$ crystal the peaks correspond to transitions from the first spin-orbit split band below $E_{\mathrm{F}}$ into a free electron like final band. (These transitions $\mathrm{A}$ and $\mathrm{B}$ are given in the $\mathrm{Ag}$ band structure in fig. 5.) Though the peak shift of the intensity peak between 4.3 and $4.5 \mathrm{eV}$ is also due to the decreasing Pt part in the spectra a small shift of $0.2 \mathrm{eV}$ is also observed in the $I_{+}$peak of the spectrum which purely arises from photoemission from the $\mathrm{Ag}$ adsorbate. This is probably due to the development of the three-dimensional band structure of the $\mathrm{Ag}(111)$ crystal. A $0.1 \mathrm{eV}$ shift of the peak is already observed for a coverage of 2.2 layers and increases gradually up to the final value of $0.2 \mathrm{eV}$ for the $\mathrm{Ag}(111)$ crystal.

To reveal more details of the development of the three-dimensional band structure in the $\Lambda$-direction the energy below $E_{\mathrm{F}}$ of peak $\mathrm{B}$ is plotted versus photon energy in fig. 6 for 1,3 , and 8 layers. For 1 layer the binding energy of peak B does not shift with photon energy indicating no dispersion in the $\Lambda$-direction. Strong dispersion of about $0.5 \mathrm{eV}$ is observed for both the 3 and the 8 layer system. While we get for 8 layers quantitatively the same energetic position and dispersion for peak B as expected for a $\mathrm{Ag}$ crystal (see fig. 5 and ref. [9]) the 3 layer adsorbate shows slight deviations in binding energy up to

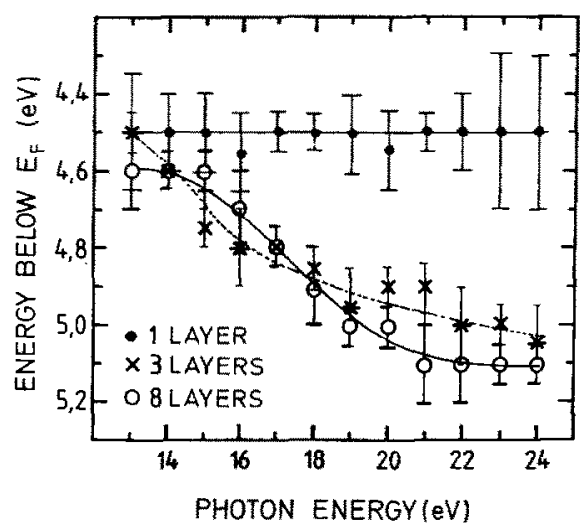

Fig. 6. Energy below $E_{\mathrm{F}}$ versus photon energy for peak $\mathrm{B}$ for 1,3 , and 8 layers. 


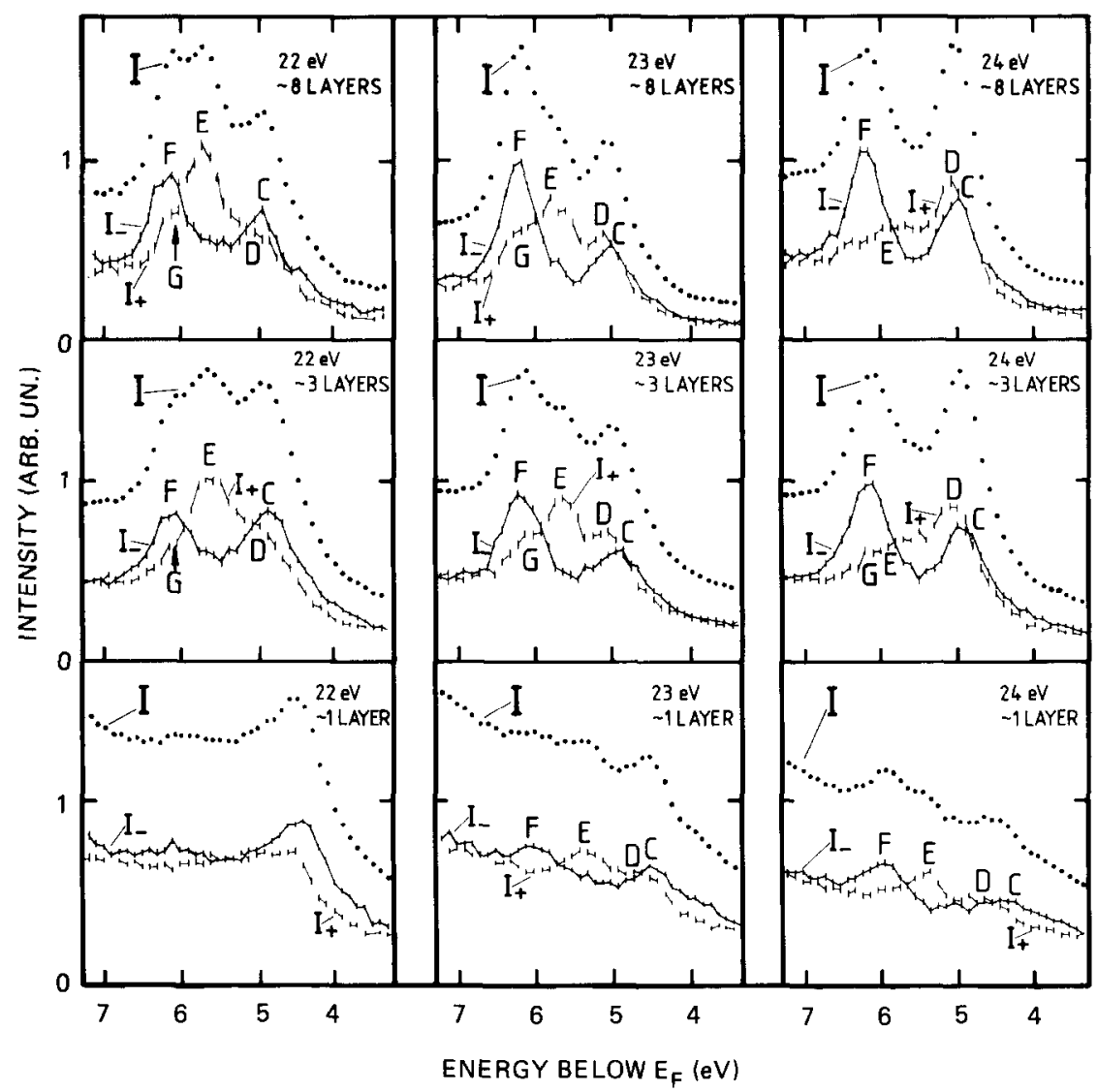

Fig. 7. Photoelectron intensities of $\mathrm{Ag} / \mathrm{Pt}(111)$ for $h \nu=22,23$, and $24 \mathrm{eV}$ and coverages of 1,3 , and 8 layers. The 4 peaks correspond to $\mathrm{Ag}$ transitions (Pt peaks are suppressed due to a bandgap in the unoccupied part of the Pt band structure). The peaks $E$ and $F$ show a stronger increase with coverage than peaks $C$ and $D$. Spin polarization reveals a further peak $G$, which is not predicted by theoretical models for the resonant behavior.

$0.2 \mathrm{eV}$. This finding supports again the conclusion that an 8 layer epitaxial $\mathrm{Ag}$ adsorbate [18] behaves in photoemission like a $\mathrm{Ag}$ crystal. The dispersion of the initial band $\mathrm{B}$ is, however, already almost completely developed for $3 \mathrm{Ag}$ layers.

The information about the development of the band structure of $\mathrm{Ag}$ obtained from the spectra in figs. 4 and 6 concerned the occupied part of the band structure. The spectra presented in fig. 7 yield also information about the development in the unoccupied part of the band structure: We have chosen the photon energies around $23 \mathrm{eV}$. For this photon energy the $\mathrm{Pt}$ intensity is drastically reduced due to a bandgap in the unoccupied part of the $\mathrm{Pt}$ band 
structure, while the Ag intensity is resonantly enhanced due to transitions into that part of the band structure where a flat f-like final band (left part of band 8 , upper part of band 7 in fig. 5) crosses the free-electron like final band (lower part of band 7 and upper right part of band 8 ). These resonances are well described in a three-step photoemission model as a combination of transition strengths calculated for an infinite three-dimensional crystal and a surface transmission factor $[9,28]$.

One step photoemission theories, which are believed [29] to offer the best hope of arriving at a fully quantitative understanding of photoemission from solid surfaces and adsorbates, do, however, not yet reproduce photoemission spectra with transitions into this part of the band structure (while the agreement of calculated and experimental spectra is generally excellent for those parts of the band structure where transitions can only occur into the free electron like final band [30]). Studies of the development of the band structure and, especially, the development of the photoemission spectra themselves with coverage in this energy range, are thus of great interest for further improvements of one-step photoemission formalisms and their extension to adsorbate systems.

Spin-resolved photoemission spectra for 1, 3, and 8 layers Ag coverage on $\mathrm{Pt}(111)$ are shown in fig. 7. The intensities $I$ for the highest coverage agrees again with spectra of a $\mathrm{Ag}$ crystal in ref. [9]. According to ref. [9] the spectra consist of 4 peaks due to transitions from the upper two spin-orbit split valence band pairs. Peaks C and D in fig. 7 correspond to transitions into the free electron like final band, while the peaks $E$ and $F$ result from transitions into the crossing region of the free electron like final band and the flat f-like band. Spin analysis reveals a further shoulder at the low energy side of peak E, which we denote by $G$. Peak $G$ shows also a resonant behavior with photon energy as the peaks $E$ and $F$, but we do not observe a corresponding shoulder in $I_{-}$at the low energy side of peak $F$.

Searching for an explanation for the occurrence of peak $G$ we discuss some features in non-spin-resolved and spin-resolved photoemission spectra obtained in the past by different authors and which could not be attributed to direct transitions into a free electron like final band. In a non-spin-resolved study on $\mathrm{Au}(111)$ Courths et al. [11] found dispersionsless structures D1, D2 and D3, which could not be identified unambiguously and were tentatively be interpreted as surface resonances. As our peak $G$ these structures depend resonantly on photon energy but our peak $G$ seems to show a slight dispersion, namely a shift towards higher binding energy when going from 22 to $23 \mathrm{eV}$ photon energy in fig. 7 . In addition peak $\mathrm{G}$ increases with Ag coverage as can be seen from a comparison of the spin-resolved photoemission spectra for different coverages in fig. 7. This is a finding which causes us to search an explanation for peak $G$ in terms of the three-dimensional band structure of Ag. In a very recent work [30] Tamura, Piepke and Feder pointed out that by 
means of the spin-resolved photoemission technique it is possible to resolve further peaks, the so-called "ghost peaks", in the photoemission spectra. Ghost peaks arise from spin flip processes and occur thus at the same binding energy as a main direct transition peak but in the opposite spin part. Peak G can thus not be a ghost peak, as peak $G$ is located at a binding energy different from those of the two direct transitions $E$ and $F$. While peak $G$ is thus obviously not due to a direct transition into the free electron like part of the final bands it is energetically possible that peak $\mathrm{G}$ arises from a transition into a second final band. That such transitions exist and contribute to photoemission spectra has been verified by Müller et al. [5] in a spin-resolved photoemission study on $\operatorname{Ir}(111)$. One of these transitions could also be identified in a work on $\mathrm{Pt}(111)$ by Garbe et al. [31]. Because of its energetic position peak $G$ can (in terms of the band structure) be explained by a transition from the avoided crossing region of the lower occupied $\Lambda_{6}^{3}$-band with the $\Lambda_{6}^{1}$-band. This is especially supported by the fact that we do not observe a corresponding shoulder in the opposite spin part of the spectrum at the low energy side of peak F. One should also note, however, that transitions from this part of the band structure are not predicted by the simple model in refs. $[9,28]$, which describes the general behavior of the resonances quite well. Theoretical as well as even more detailed experimental studies (also with other substrates) seem thus to be required for an explicit proof and understanding of the nature of peak $G$ and a further understanding of the resonances.

\section{Summary}

We studied the system $\mathrm{Ag} / \mathrm{Pt}(111)$ with spin-, angle-, and energy-resolved photoemission. Ag coverages between 0 and 8 layers grew up epitaxially on the $\mathrm{Pt}(111)$ crystal in an almost layer-by-layer fashion as could be shown by a combined study with LEED/Auger, photoemission and RBS/channeling. Spin-resolved photoemission data for the different coverages gave information about the development of both the occupied and the unoccupied part of the band structure that cannot be obtained without spin analysis. $\Lambda$ comparison of the energy below $E_{\mathrm{F}}$ depending on the photon energy for one peak for 1, 3, and 8 layers showed that a 3 layer epitaxially grown $\mathrm{Ag}$ adsorbate behaves in photoemission nearly as a $\mathrm{Ag}$ crystal. The resonant behavior of the peak with highest binding energy is also already observed for a 3 layer $\mathrm{Ag}$ adsorbate. Spin analysis shows that the resonances contain also a peak which seems to be due to a transition, that is not predicted by the model given in refs. $[9,28]$.

\section{Acknowledgements}

We would like to thank Professor H.O. Lutz for making available to us the Rutherford backscattering facilities. Discussions with N. Müller are gratefully 
acknowledged. Thanks are also due to $\mathrm{E}$. Tamura and R. Feder for discussions and for providing data (ref. [30]) prior to publication as well as to our colleagues of the BESSY staff for their support. The work was supported by the Bundesministerium für Forschung und Technologie (05 331 AXI).

\section{References}

[1] U. Heinzmann, Phys. Scripta $T 17$ (1987) 77, and references therein.

[2] G. Schönhense, Appl. Phys. A 41 (1986) 39, and references therein.

[3] A. Eyers, F. Schäfers, G. Schönhense, U. Heinzmann, H.P. Oepen, K. Hünlich, J. Kirschner and G. Borstel, Phys. Rev. Letters 52 (1984) 1559;

H.P. Oepen, K. Hünlich, J. Kirschner, A. Eyers, F. Schäfers, G. Schönhense and U. Heinzmann, Phys. Rev, B 31 (1985) 6846.

[4] B. Schmiedeskamp, B. Kessler, N. Müller, G. Schönhense and U. Heinzmann, Solid State Commun. 65 (1988) 665.

[5] N. Müller, B. Kessler, B. Schmiedeskamp, G. Schönhense and U. Heinzmann, Solid State Commun. 61 (1987) 187.

[6] B. Kessler, A. Eyers, K. Horn, N. Müller, B. Schmiedeskamp, G. Schönhense and U, Heinzmann, Phys. Rev. Letters 59 (1987) 331.

[7] P.W. Davies, M.A. Quinlan and G.A. Somorjai, Surface Sci. 121 (1982) 290.

[8] M. Salmerón, S. Ferrer, M. Jazzar and G.A. Somorjai, Phys. Rev. B 28 (1983) 6758.

[9] M. Wern, R. Courths, G. Leschik and S. Hüfner, Z. Phys. B 60 (1985) 293.

[10] C.M. Schneider, J. Garbe, K. Bethke and J. Kirschner, Phys. Rev. B 39 (1989) 1031.

[11] R. Courths, H.-G. Zimmer, A. Goldmann and H. Saalfeld, Phys. Rev. B 34 (1986) 3577.

[12] U. Lipphardt, H. Engelhard, A. Goldmann and H. Saalfeld, BESSY-Jahresbericht (1987) p. 250.

[13] R. Allenspach, F. Meier and D. Pescia, Phys. Rev. Letters 51 (1983) 2148;

J. Garbe, D. Venus, S. Suga, C. Schneider and J. Kirschner, Surface Sci. 178 (1986) 342.

[14] F. Schäfers, W. Peatman, A. Eyers, Ch. Heckenkamp, G. Schönhense and U. Heinzmann, Rev. Sci. Instrum. 57 (1986) 1032.

[15] K. Jost, J. Phys. E 12 (1979) 1001, 1006.

[16] G.E. Rhead, J. Vacuum Sci. Technol. 13 (1976) 603.

[17] Note that we also assume a constant sticking probability versus coverage as the Ag dosage versus time is constant. The constance of $\mathrm{Ag}$ dosage versus time was tested by reproducing the AST plot of fig. 1 several times.

[18] As shown above the data are compatible with a layer-by-layer growth. The AST plot does, however, not explicitly prove this, as breaks could not clearly be revealed in the data. It can thus not be excluded that small amounts of $\mathrm{Ag}$ start to buildup a new layer before the underlying layer is completed. Extremely flat epitaxial islands are also compatible with the AST plot. They should, however, cover almost the whole Pt substrate as is also demonstrated by the reduction of the $\mathrm{Pt}$ signal in the photoemission spectra in fig. 5 .

[19] B. Schmiedeskamp, P. Jonk, H.E. Roosendaal and H.O. Lutz, Nucl. Instr. Methods B 17 (1986) 309

[20] The $\mathrm{Ag}$ yield is so low both due to a lower backscattering cross section (which scales with the square of the target atom charge number $Z_{2}$ ) and due to the thickness of the Ag layer which is by about a factor of 10 thinner than the experimental depth resolution.

[21] I. Stensgaard, L.C. Feldman and P.J. Silverman, Surface Sci. 77 (1978) 513.

[22] While the random spectrum was collected, the sample was rotated by $120^{\circ}$ to take into account the threefold symmetry of $\mathrm{Pt}(111)$. A similar procedure is proposed in: J.F. Ziegler, B.L. Crowden, Appl. Phys. Letters 20 (1972) 178. 
[23] The value of 1.1 ML is a Monte Carlo value taken from ref. [21]. Experimental values seem to be slightly larger (see e.g. fig. 8 in ref. [17]). The $1.1 \mathrm{ML}$ consist of $1 \mathrm{ML}$ for backscattering from the first layer and almost $0.1 \mathrm{ML}$ from the second layer.

[24] For a $\mathrm{Ag}$ atom on top of a $\mathrm{Pt}$ string the screening will be less effective than for a pure $\mathrm{Pt}$ string as Ag has a lower charge number and probably a larger thermal vibration amplitude. A value of about $0.5 \mathrm{ML}$ for backscattering from the first $\mathrm{Pt}$ atom behind the Ag atom (as also expected for the second atom in a pure $\mathrm{Ag}$ string) seems to be realistic.

[25] Note that the screening of the Pt sublattice is largely reduced compared to what is experimentally observed, if the $\mathrm{Ag}$ atoms are displayed by more than the shadow cone radius $R_{\mathrm{M}}$ of about $0.2 \AA$.

[26] E. Tamura, R. Feder, B. Vogt, B. Schmiedeskamp and U. Heinzmann, to be published.

[27] H. Eckhard, L. Fritsche and J. Noffke, J. Phys. F (Met. Phys.) 14 (1984) 97.

[28] R.L. Benbow and N.V. Smith, Phys. Rev. B 27 (1983) 3144.

[29] N.V. Smith, in: Photoemission in Solids I, Eds. M. Cardona and I. I ey (Springer, Rerlin, 1978) p. 254.

[30] E. Tamura, W. Piepke and R. Feder, J. Phys. (Cond. Matter) 1 (1989) 6469.

[31] J. Garbe and J. Kirschner, Phys. Rev. B 39 (1989) 1530. 